\title{
HYDRO-ELASTIC MODELLING OF AN ELECTRO-ACTIVE WAVE ENERGY CONVERTER
}

\author{
Aurélien Babarit \\ LUNAM Université \\ Ecole Centrale De Nantes - CNRS \\ Nantes, France
}

\author{
Benjamin Gendron \\ LUNAM Université \\ Ecole Centrale De Nantes - CNRS \\ Nantes, France
}

\author{
Jitendra Singh \\ LUNAM Université \\ Ecole Centrale De Nantes - CNRS \\ Nantes, France
}

\author{
Cécile Mélis \\ SBM offshore \\ Monaco
}

\author{
Philippe Jean \\ SBM offshore \\ Monaco
}

\section{ABSTRACT}

Since 2009, SBM Offshore has been developing the S3 Wave Energy Converter (S3 WEC). It consists in a long flexible tube made of an Electro-Active Polymer (EAP). Thus, the structural material is also the Power Take Off (PTO).

In order to optimize the S3 WEC, a hydro-elastic numerical model able to predict the device dynamic response has been developed. The inner flow, elastic wall deformations and outer flow are taken into account in the model under the following assumptions: Euler equation is used for the inner flow. The flow is also assumed to be uniform. Elastic deformation of the wall tube is linearized. The outer flow is modeled using linear potential theory. These equations have been combined in order to build the numerical model. First, they are solved in the absence of the outer fluid in order to obtain the modes of response of the device. Secondly, the outer fluid is taken into account and the equation of motion is solved by making use of modal expansion.

Meanwhile, experimental validation tests were conducted in the ocean basin at Ecole Centrale De Nantes. The scale model is $10 \mathrm{~m}$ long tube made of EAP. The tube deformations were measured using the electro-active polymer. The model was also equipped with sensors in order to measure the inner pressure. Comparisons of the deformation rate between the numerical model and experimental results show good agreement, provided that the wall damping is calibrated.

Eventually, results of a technico-economical parametric study of the dimensions of the device are presented.

\section{INTRODUCTION}

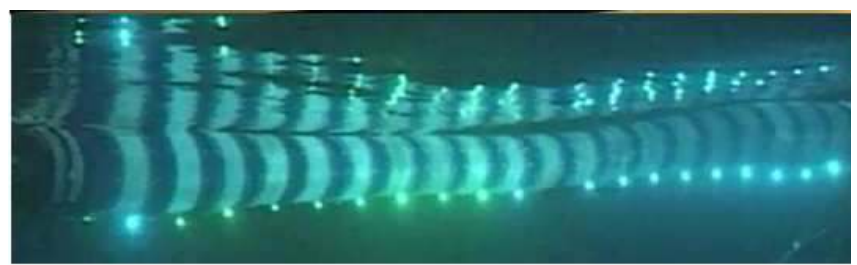

Figure 1. Underwater picture of the scale model of the S3

WEC during experiments at Ecole Centrale De Nantes.

Since 2009, SBM offshore has been developing a radically new wave energy converter named S3 [1]. It consists in a long flexible tube which is made of an electro-active polymer. Thus, the structural material is also the power take off. A picture of a scale model of the device taken during experiments at Ecole Centrale de Nantes is shown in Figure 1. 


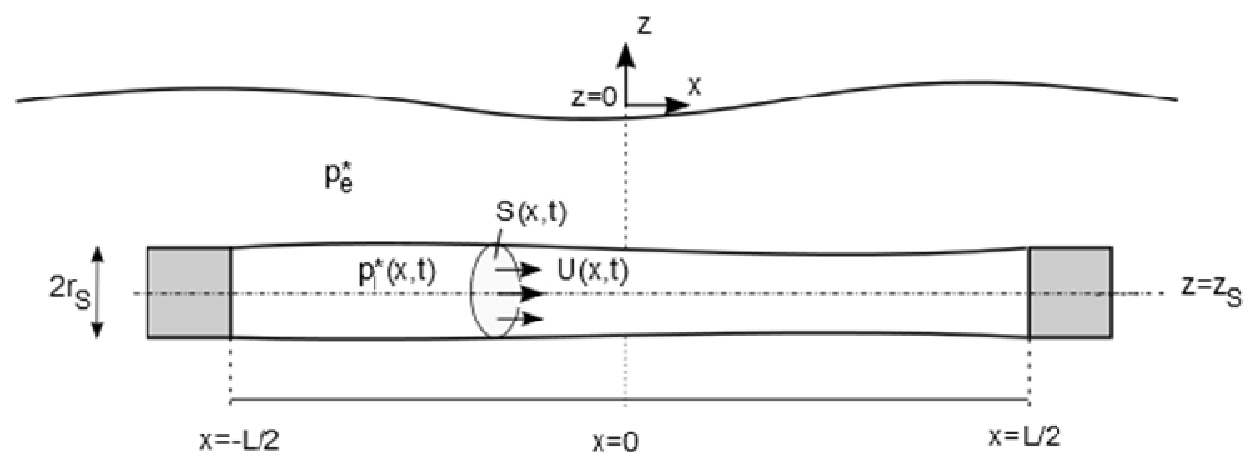

Figure 2. Notations

The working principle of the S3 WEC is as follow: The tube is filled with sea water slightly pressurized. It is floating right beneath the free surface. Under wave action, the tube deforms: bulge waves are generated on the surface together with pressure waves inside the tube. They propagate with their own velocities depending on the dimensions and properties of the material. It results in a pressure difference between the inner and outer surface of the tube. The work done by the pressure difference and the deformation of the tube wall is the wave energy which is absorbed by the device. The EAP material converts the absorbed energy into electricity by change of the electric capacitance due to the wall thickness variations associated with the radial deformations.

In comparison with other WEC technologies, the S3 WEC has many advantages. The most important ones are:

- The PTO is also the structure of the device. Thus, fabrication costs of the structure are avoided. That is why the system is expected to be cost competitive with respect to other conventional WECs (heaving buoys, OWCs, overtopping devices...).

- The PTO is distributed over the whole surface of the device. It allows avoiding stress concentration which is the source of many reliability issues in conventional WECs.

- In survival conditions, the WEC can be deflated. It results in a long compliant structure which would not have to deal with extreme loadings.

In the first section of this paper, a numerical model of the device able to predict the dynamic response of the device and its energy production is presented. Experimental validations are presented in the second section. Eventually, results of a technico-economical parametric study of the dimensions of the device are presented.

\section{NUMERICAL MODEL}

In order to optimize the dimensions and parameters of the system, SBM and Ecole Centrale De Nantes have been developing in collaboration a numerical Wave to Wire (W2W) model able to predict the dynamic response of the S3 WEC.
The methodology is summarized in what follows. More details can be found in [2].

\subsection{Notations and assumptions}

The length of the tube is denoted $L$. It is made of an elastic electro active material of density $\rho_{\text {tube }}$. The radius at equilibrium is denoted $r_{S} . z_{S}$ is the vertical coordinate of the axis and $h_{S}$ is the wall thickness which is considered small with respect to $r_{s}$. The tube mass can be approximated by $M_{\text {tube }}=2 \rho_{\text {tube }} \pi r_{S} h_{S} L$.

Let assume that the bulge deformation is homogeneous in the radial direction. Thus, it depends only on the horizontal coordinate $x$ and on the time $t$. Let denote $r(x, t)$ the radius et $S(x, t)$ the cross section.

The tube being reinforced in the horizontal direction with rigid fibers, let assume that it cannot be deformed in that direction. Therefore, the surge motion is a rigid body motion which is denoted $X$.

Both ends of the tube are embedded in a rigid tow-head, with mass $M_{\text {towhead }}$. Thus the tube cross-sections at both extremities are $S(x=-L / 2)=S(x=L / 2)=S_{S}, S_{S}$ being the area of the cross section at static equilibrium. Each tow-head is connected to a mooring system.

In what follows, it is assumed that all dynamic quantities are small enough to allow linearization.

\subsection{Governing equations}

- Inner flow: It is assumed that viscosity effects are negligible for the inner flow and that the flow is homogeneous radially (the pressure and velocity depends only on the $\mathrm{x}$ coordinate and on time).The equations governing the inner flow result from Euler equation and mass conservation yield:

$$
\left\{\begin{array}{l}
\frac{\partial U}{\partial t}=-\frac{1}{\rho} \frac{\partial p_{i}}{\partial x} \\
\frac{\partial S}{\partial t}=-S_{S} \frac{\partial U}{\partial x}
\end{array}\right.
$$


With $U(x, t)$ the flow velocity in the tube and $p_{i}$ the dynamic part of the inner pressure. $\rho$ is the water density.

- Outer flow: The dynamic part of the outer pressure is denoted $p_{e}$. Let define $\bar{p}_{e}(x, t)$ its average over the external contour $C(x)$ of the tube.

The outer flow is modeled within the frame of linear potential flow theory. Thus, the components of the outer flow (diffraction, radiation) can be separated. Pressure loads associated with diffraction are obtained using standard approach [3]. For radiation loads, generalized mode approach is employed [4]: the boundary value problem is solved for each mode shape (see section 2.3) and the total radiation load is obtained by linear superposition.

- Wall equation: The wall equation is obtained by considering the inner and outer forces applying on a small piece of the tube wall and averaging on the contour. One can show:

$$
p_{i}=\bar{p}_{e}-\frac{T_{S}}{4 \pi S_{S}} \frac{\partial^{2} S}{\partial x^{2}}+\frac{h_{S}}{r_{S}}\left(\frac{S_{S}}{S} \sigma_{\theta}-\sigma_{\theta_{S}}\right)
$$

With $T_{S}$ the wall pretension in the longitudinal direction and $\sigma_{\theta}(x, t)$ the wall constraint ( $\sigma_{\theta_{S}}$ being its value at equilibrium).

Let assume that the difference $\left(\frac{S_{S}}{S} \sigma_{\theta}-\sigma_{\theta_{S}}\right)$ can be modeled as a linear spring and damper whose coefficients are $K$ and $B$. The linearised wall equation can be rewritten:

$$
p_{i}=\bar{p}_{e}-\frac{1}{D S_{S}} \frac{1}{K_{0}^{2}} \frac{\partial^{2} S}{\partial x^{2}}+\frac{1}{D S_{S}}\left(S-S_{S}\right)+\frac{\rho}{S_{S}} \eta \dot{S}
$$

With $D=\frac{r_{S}}{h_{S} S_{S} K}, \frac{1}{K_{0}^{2}}=\frac{T_{S} D}{4 \pi}$ and $\eta=\frac{h_{S} S_{S} B}{\rho r_{S}}$.

$D$ is called the distensibility [5].

By looking at equation (3), one can see that the pressure difference between the outer and the inner surfaces of the tube is the sum of a bending stiffness, an elastic stiffness and a linear damping term.

- Horizontal motion: It is assumed that the tube cannot be deformed horizontally because of the reinforcing fibers. Thus, the surge motion of the tube is a rigid body motion. By applying Newton's law to each towhead, by integrating Euler's equation over the length of the tube and by adding all these three equations, one can show:

$$
M \ddot{X}=-2 K_{a} X+\left(\Delta p_{i}-\Delta \bar{p}_{e}\right) S_{S}
$$

With $M=M_{\text {tube }}+2 M_{\text {towhead }}, \quad K_{a}$ is the horizontal mooring stiffness, $\Delta p_{i}=\left.p_{i}\right|_{x=\frac{L}{2}}-\left.p_{i}\right|_{x=-\frac{L}{2}}$ is the inner pressure difference between the both ends of the tube and $\Delta \bar{p}_{e}=\left.\bar{p}_{e}\right|_{x=\frac{L}{2}}-\left.\bar{p}_{e}\right|_{x=-\frac{L}{2}}$ is the outer pressure difference.

Equation (4) shows that the surge motion is governed by the mooring stiffness and the inner and outer pressure difference at both ends of the tube.

- Boundary conditions: The tube is embedded in the tow-heads at both ends. Thus, the tube cannot deform at its ends. In addition, the tube being closed, the inner flow velocity at the tube ends must be equal to the tube horizontal velocity. Mathematically, these conditions read:

$$
\left\{\begin{array}{l}
S\left( \pm \frac{L}{2}\right)=S_{S} \\
U\left( \pm \frac{L}{2}\right)=\dot{X}
\end{array}\right.
$$

\subsection{Mode shapes}

Let us look for the mode shapes of the tube (free oscillations in calm water $\bar{p}_{e}=0$ without damping $B=0$ ). Radiation effects are not taken into account.

Let us define a complimentary variable $\chi$ such as its time derivative is equal to the inner fluid velocity:

$$
\frac{\partial \chi}{\partial t}=U
$$

By using the second equation in (1), integrating over time and assuming that the tube is initially at rest, one can show that the relative deformation $\left(S-S_{S}\right)$ is related to the complimentary variable $\chi$ :

$$
\left(S-S_{S}\right)=-S_{S} \frac{\partial \chi}{\partial x}
$$

Thus, by combining equations (1), (3) and (7), one can obtain the propagation equation:

$$
\frac{\partial^{2} \chi}{\partial t^{2}}-\frac{1}{\rho D} \frac{\partial^{2} \chi}{\partial x^{2}}+\frac{1}{\rho D} \frac{1}{K_{0}^{2}} \frac{\partial^{4} \chi}{\partial x^{4}}=0
$$

With the associated boundary conditions are obtained by combining (5), (6) and (7): 


$$
\left\{\begin{array}{l}
\frac{\partial \chi}{\partial x}\left( \pm \frac{L}{2}\right)=0 \\
\frac{\partial \chi}{\partial t}\left( \pm \frac{L}{2}\right)=\dot{X}
\end{array}\right.
$$

By using standard techniques for separating variables, the boundary conditions (9) and the surge motion equation (4), one can show that the solutions of (9) read $\chi(x, t)=\tilde{\chi}_{n}^{(k)}(x) e^{i \omega_{n}^{(k)} t}$, $k \in\{1,2\}$ with:

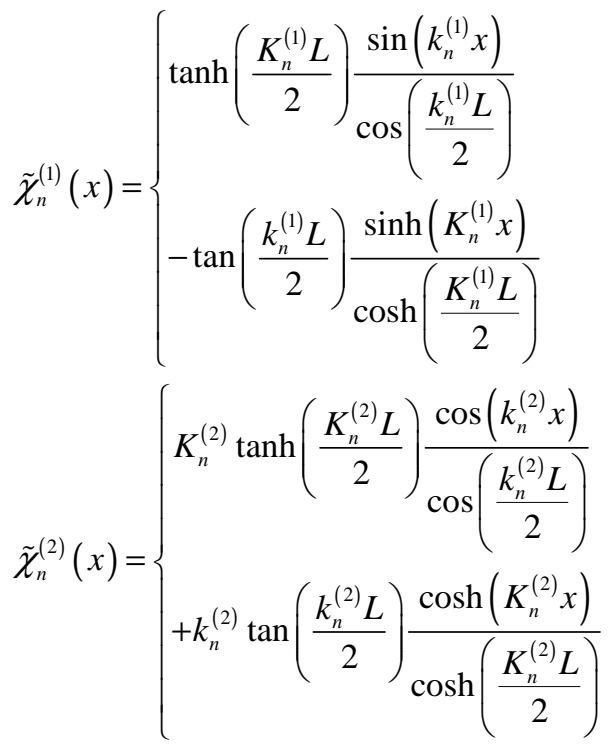

The wave numbers $K_{n}^{(1)}, k_{n}^{(1)}, K_{n}^{(2)}, k_{n}^{(2)}$ are the solutions of the wave equations:

$$
\begin{aligned}
& \frac{k_{n}^{(1)} L}{2} \tanh \left(\frac{K_{n}^{(1)} L}{2}\right)=\frac{K_{n}^{(1)} L}{2} \tan \left(\frac{k_{n}^{(1)} L}{2}\right) \\
& \left\{\begin{array}{l}
\frac{K_{n}^{(2)} L}{2} \tanh \left(\frac{K_{n}^{(2)} L}{2}\right)+\frac{k_{n}^{(1)} L}{2} \tan \left(\frac{k_{n}^{(1)} L}{2}\right) \\
+\frac{\rho S_{S} L\left(\omega_{n}^{(1)}\right)^{2}}{M\left(\omega_{n}^{(1)}\right)^{2}-2 K_{a}}\left(\frac{K_{n}^{(1)}}{k_{n}^{(1)}}+\frac{k_{n}^{(1)}}{K_{n}^{(1)}}\right) \tanh \left(\frac{K_{n}^{(2)} L}{2}\right) \tan \left(\frac{k_{n}^{(2)} L}{2}\right)
\end{array}\right.
\end{aligned}
$$

The natural frequencies $\omega_{n}^{(k)}$ can be obtained by applying equation (8) to each mode $\tilde{\chi}_{n}^{(k)}(x) e^{i \omega_{n}^{(k)} t}$.
To simplify the notations in what follows, let us define the set of modal responses $\hat{\chi}_{m}$ corresponding with the mode shapes $\tilde{\chi}_{n}^{(k)}(x)$ ordered by their modal frequencies.

Figure 3 shows the four first mode shapes $\left(S-S_{S}\right)_{n}^{(k)}$ for the case of the $200 \mathrm{~m}$ long tube of diameter $3 \mathrm{~m}$. They have been obtained by applying equation (7) to each mode $\tilde{\chi}_{n}^{(k)}(x)$. As expected, the mode shapes look like sine or cosine functions with increasing number of oscillations as the mode number increases. However, one can observe the rapid change in the section close to both ends of the tube due to the embedment condition of the tube in the tow-heads.

\subsection{Forced oscillations}

Let's look for the complete solution of the problem, i.e. the tube motion is forced by the waves and the radiation effects and linear damping (energy absorption) in the electro-active material are taken into account. The corresponding propagation equation is:

$$
\frac{\partial^{2} \chi}{\partial t^{2}}-\frac{1}{\rho D} \frac{\partial^{2} \chi}{\partial x^{2}}+\frac{1}{\rho D} \frac{1}{K_{0}^{2}} \frac{\partial^{4} \chi}{\partial x^{4}}=-\frac{1}{\rho} \frac{\partial \bar{p}_{e}}{\partial x}+\eta \frac{\partial^{2} \dot{\chi}}{\partial x^{2}}
$$

The solution is written under the form of a linear combination of the mode shapes whose coefficients depend on time:

$$
\chi(x, t)=\sum_{m} c_{m}(t) \hat{\chi}_{m}(x)
$$

By using this expression in (12), by manipulating the equations and by defining the model projection $\chi_{f}$ of any function $f$ such as:

$$
{ }^{\chi} f=\frac{1}{\rho} \int_{-\frac{L}{2}}^{\frac{L}{2}} f(x) \frac{\partial \hat{\chi}_{m}}{\partial x} d x-\left[\left.\left(f \hat{\chi}_{m}\right)\right|_{x=\frac{L}{2}}-\left.\left(f \hat{\chi}_{m}\right)\right|_{x=-\frac{L}{2}}\right] .
$$

one can show that the coefficients $\mathbf{C}=\left[c_{m}\right]_{1 \leq m \leq N}$ are solutions of the differential matrix equation (in frequency domain):

$$
\left(\mathbf{I}+{ }^{\chi} \mathbf{A}\right) \ddot{\mathbf{C}}+\left({ }^{\chi} \mathbf{B}+\eta \boldsymbol{\varepsilon}\right) \dot{\mathbf{C}}+\omega_{m}{ }^{2} \mathbf{C}={ }^{\chi} \mathbf{p}_{\mathrm{ex}}
$$

$=0 \quad$ With $\mathbf{I}$ the identity matrix, ${ }^{\boldsymbol{x}} \mathbf{A}$ the added mass matrix of the mode shapes, $\boldsymbol{x}_{\mathbf{B}}$ the radiation damping matrix, $\varepsilon=\left(\int_{-\frac{L}{2}}^{\frac{L}{2}} \frac{\partial \hat{\chi}_{m}}{\partial x} \frac{\partial \hat{\chi}_{l}}{\partial x} d x\right)_{1 \leq l \leq N, 1 \leq m \leq N}$ the modal damping matrix and ${ }^{x} \mathbf{p}_{\mathbf{e x}}$ the column vector of the modal excitation coefficients (due to the incident and diffracted waves).

Equation (14) can be easily solved by standard matrix inversion techniques. The solution gives access to the dynamic response of the system to various wave conditions and thus to estimates for the energy production. 

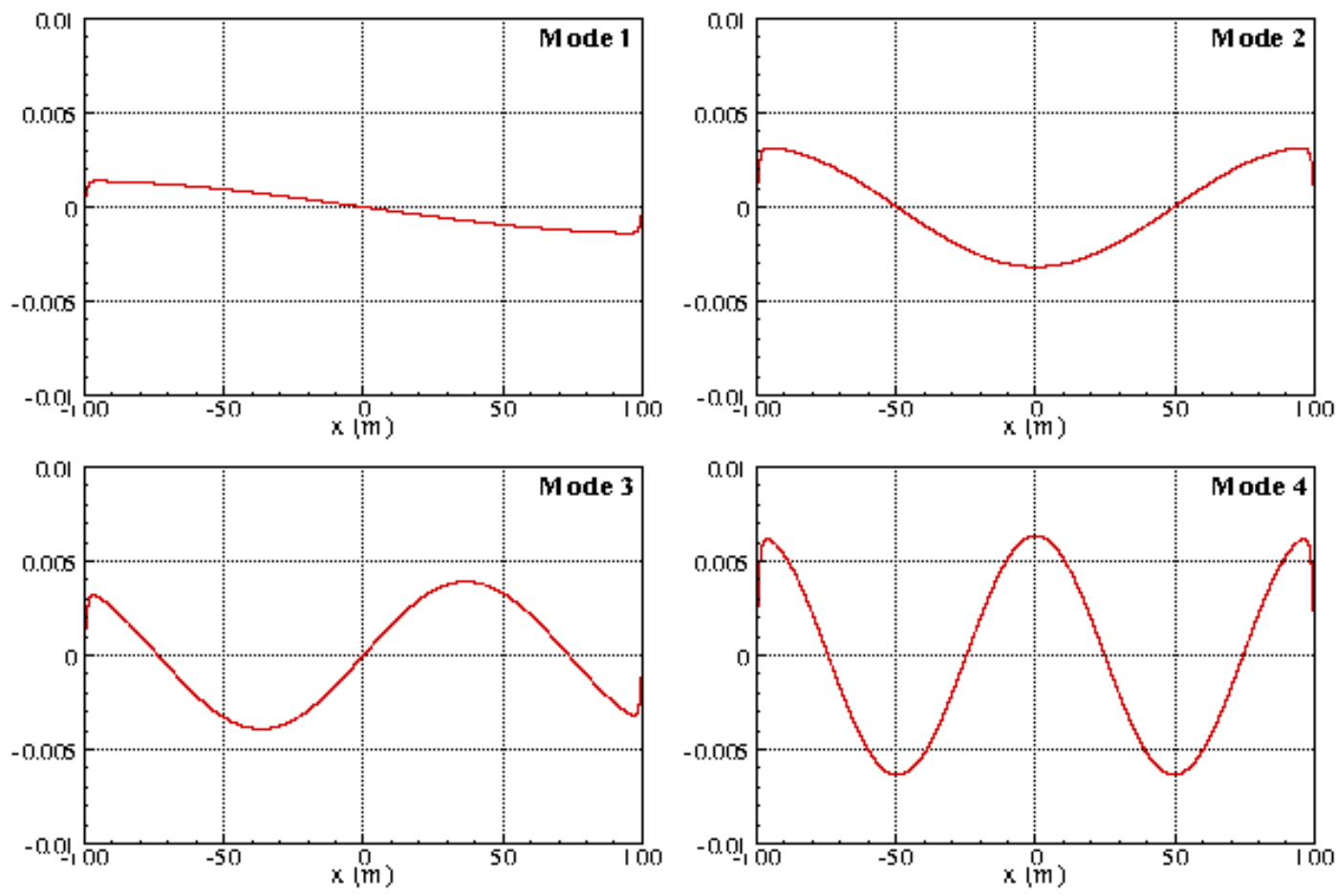

Figure 3. Four first mode shapes for the radial deformation $\left(S-S_{S}\right)$ of a $200 \mathrm{~m}$ long tube with diameter $3 \mathrm{~m}$.

\section{EXPERIMENTAL VALIDATION}

Tests have been carried out in July 2011 in the Hydrodynamics and Ocean Engineering Tank of Ecole Centrale de Nantes in order to validate the numerical model. The experimental model is a $10 \mathrm{~m}$ long tube made of silicone and covered with electro-active polymer sections. The tube deformations were measured using the electro-active material. The inner pressure was measured with sensors distributed along the tube.

\subsection{Experimental Model and instrumentation}

The experimental model was made of the following elements:

- A main silicon tube, closed at both ends, filled with water, reinforced with Aramid fibers in the longitudinal direction to avoid axial deformations.

- 20 independent sections made of multiple layers of electro-active polymer encapsulated in a silicone shell and waterproof electric connections to wire every section. These sections were slipped onto the main tube (light stripes in Figure 1 and Figure 4) and kept in place due to the frictional effect once the tube is inflated.

- Silicon separations to hold in place the wire connections (dark blue stripes on Figure 1 and Figure 4)

- Two inflatable plugs to seal the main tube at both ends, to fill up the inner chamber and transfer the mooring tensions.
- Foam pads inserted inside the tube to provide the necessary buoyancy to keep the system afloat. .

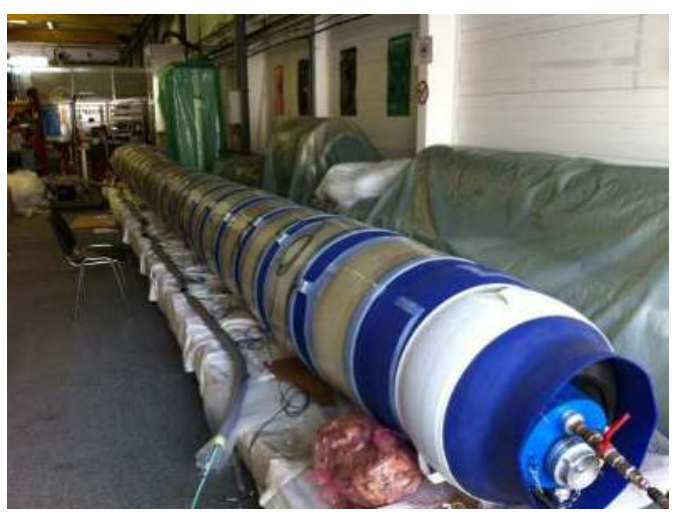

Figure 4. Experimental model during assembly - inflating test.

The deformable element of the experimental model is $10 \mathrm{~m}$ long for the first part of the tests and $7 \mathrm{~m}$ long for the second part. The diameter at rest (not inflated) is $0.42 \mathrm{~m}$.

The experimental model is moored at the center of the basin by means of 4 anchor lines set nearly horizontal and attached to springs. The entire system presents a surge motion (if the system was a rigid body) period which is much higher than the one of the tested waves (around 15s for the $10 \mathrm{~m}$ long tube).

In order to validate the numerical model, the following parameters were measured:

- The tube radial deformations for every EAP sections. Indeed, every layers of EAP have the 
Table 1 : Test series done during model test at ECN in July 2011

\begin{tabular}{|c|c|c|c|c|c|}
\hline Length [m] & Inflation ratio [\%] & Heading [deg] & Type & $\begin{array}{l}\text { Significant wave } \\
\text { height }[\mathrm{m}]\end{array}$ & Wave Period $T_{p}[\mathrm{~s}]$ \\
\hline 10 & 30 & 0 & regular & 0.2 & $\begin{array}{l}1 \text { to } 3 \text { (by steps } 0.1 \mathrm{~s} \text { ) } \\
3 \text { to } 4 \text { (by steps } 0.25 \mathrm{~s} \text { ) } \\
4 \text { to } 5 \text { (by steps } 0.5 \mathrm{~s} \text { ) }\end{array}$ \\
\hline 10 & 30 & 0 & irregular & 0.2 & $3.2 ; 4.5$ \\
\hline 10 & 30 & 0 & regular & 0.4 & $\begin{array}{l}2.2 \text { to } 3 \text { (bys steps } 0.1 \mathrm{~s} \text { ) } \\
3 \text { to } 4 \text { (by steps } 0.25 \mathrm{~s} \text { ) } \\
4 \text { to } 5 \text { (by steps } 0.5 \mathrm{~s} \text { ) }\end{array}$ \\
\hline 7 & 30 & 0 & regular & 0.2 & $\begin{array}{l}2.6 \text { to } 3 \text { (by steps } 0.1 \mathrm{~s}) \\
3 \text { to } 3.75 \text { (by steps } 0.25 \mathrm{~s} \text { ) }\end{array}$ \\
\hline 7 & 30 & 0 & regular & 0.1 & $\begin{array}{l}1.4 \text { to } 3 \text { (by steps } 0.1 \mathrm{~s}) \\
3 \text { to } 3.75 \text { (by steps } 0.25 \mathrm{~s} \text { ) }\end{array}$ \\
\hline 7 & 30 & 30 & regular & 0.1 & $\begin{array}{l}1.1 \text { to } 3 \text { (by steps } 0.1 \mathrm{~s} \text { ) } \\
3 \text { to } 3.75 \text { (by steps } 0.25 \mathrm{~s} \text { ) }\end{array}$ \\
\hline 7 & 30 & 0 & irregular & 0.1 & 2.4 \\
\hline 7 & 30 & 30 & irregular & 0.1 & $1.4 ; 1.8 ; 2.4$ \\
\hline 7 & 20 & 0 & regular & 0.1 & $\begin{array}{l}1.4 \text { to } 3 \text { (by steps } 0.1 \mathrm{~s} \text { ) } \\
3 \text { to } 3.75 \text { (by steps } 0.25 \mathrm{~s} \text { ) }\end{array}$ \\
\hline 7 & 40 & 0 & irregular & 0.2 & $1.8 ; 2.4 ; 3.2$ \\
\hline
\end{tabular}

property to change its capacitance when it is subject to deformations. For a considered deformation, the capacitance is given by the following relation: $C=C_{0} \lambda^{p}$ with $C$ the capacitance under constraint, $C_{0}$ the capacitance at rest and $\lambda$ the hoop elongation ratio, ratio between the deformed radius and the radius at rest, and $p$, a form factor. Every section had been previously calibrated and the values $C_{0}$ and $p$ defined. During the tests, the sections were wired to a capacitance meter yielding the instant $\lambda$ values along the tube.

- The inner and outer pressures along the tube. Two straps with 20 ceramic membrane pressure sensors were assembled. One was set inside the main tube chamber and the other was set on the outside of the tube. Magnets were stitched onto the straps in order to attach the two in opposition on both sides of the tube.

- The point's 3D coordinates underneath the experimental model (light points on figure 1) with trajectography cameras. A "tinsel" of LEDs (active targets) was fixed underneath the experimental model using the same magnets that were used to attach the straps. Six underwater cameras were disposed on the basin bottom in order to record the trajectory of the targets and adhoc software was used to calculate the target's position.
- The four anchor lines tensions with force sensors.

- Wave height at different positions around the experimental model as well as the undisturbed elevation at the center of the experimental model measured at the beginning of the tests.

\subsection{Wave tests}

Table 1 summarizes the wave tests series done during the tests.

The tube length was modified during the model test. This variation helped visualize the effect on the eigen modes of the system.

The inflation ratio corresponds to the static deformation of the main tube. The variation of this parameter affects at the same time the mass of the system and the wall stiffness (hyperelastic behavior of the silicon), and, in the same time the eigen modes.

The different wave heights helped evaluating the importance of the non-linear terms (material heat losses, drag, etc...). The different headings confirmed that the system can operate when the swell is not aligned with the tube.

Finally, using irregular waves validated that the system can work in a real environment.

\subsection{Numerical model comparison}

Comparisons could finally be achieved between the numerical and experimental results. An overview is shown on the following figure in terms of radial deformations in relation with the wave period (vertical axis) and the tube section 
(horizontal axis). One can see a satisfactory agreement once the damping coefficient of the tube wall has been calibrated.
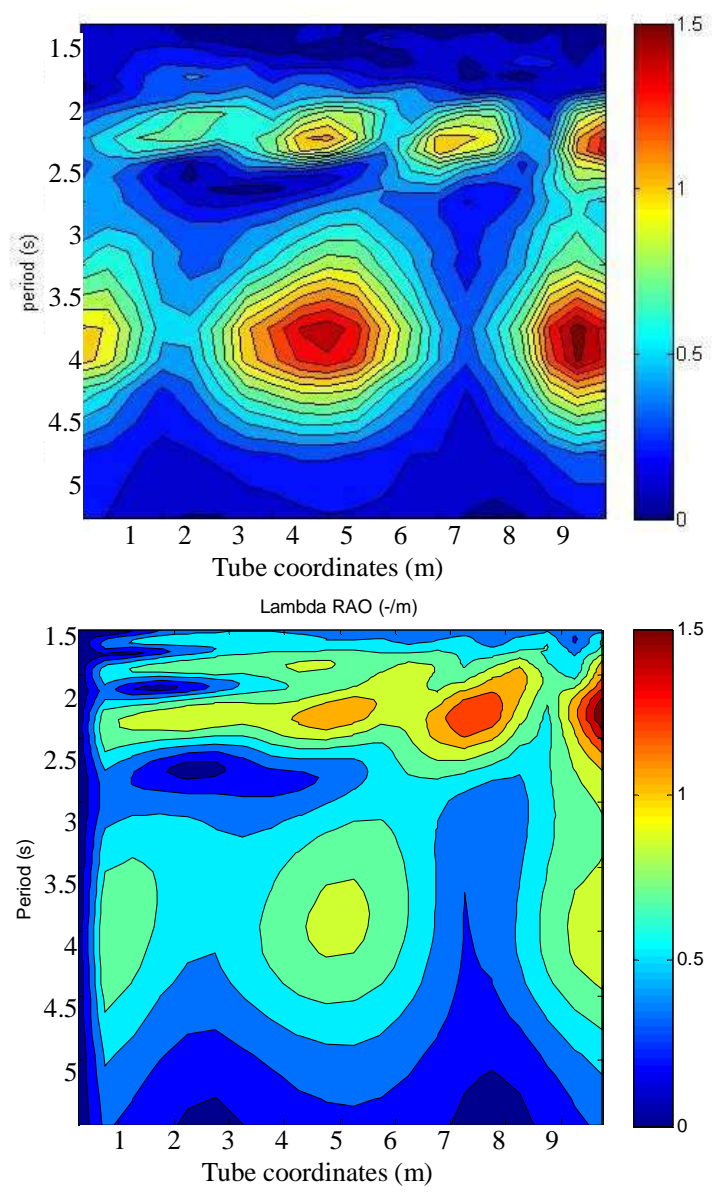

Figure 5. Comparison between experimental results (top) and numerical results (bottom) for a 10m long tube.

As the inner pressure wave is a standing wave (due to the closed ends), the large circles (yellow and red areas) corresponds to the anti-nodes where the radial deformations are important. These anti-nodes appear at two different periods which corresponds to 2 different tube shape modes. The first shape mode period is at $2.1 \mathrm{~s}$, the second at $3.6 \mathrm{~s}$ and both shape modes have different wave length. The first one presents 4 antinodes and the second one 3 . Outside these two resonance periods, the tube is barely deforming itself because there is no other deformation shape mode.

The first resonance period is due to the coupling of the inner pressure wave with the elastic wave within the tube wall and the second period is due to the coupling of the inner pressure wave with the tube surge motion.

These two resonance periods appear on both graphs. In both cases, the response is close, meaning that the $\mathrm{W} 2 \mathrm{~W}$ program can give a good prediction of the section deformations behavior. However, one can see that the $\mathrm{W} 2 \mathrm{~W}$ program predicts other modes at smaller periods (i.e. 1.6s) which were not measured in the experiments. This overestimation of the tube response at small periods is probably due to additional non linear effects which are not taken into account in the actual version of the numerical model.

\section{PARAMETRIC STUDY}

The numerical model showed sufficient agreement with the test data in this first phase and was considered validated. It was therefore further used to estimate the power output of a real system. The next phase was to find an optimum geometry in order to maximize the energy absorption with a minimum amount of active material and a satisfying fatigue life.

\subsection{Maximizing the energy absorption}

For a given configuration, the most important parameter determining the extracted energy is the wall damping coefficient $B$. This damping coefficient includes two distinct phenomena:

- The viscous losses caused by the elastomeric material which generates heat upon cycling (B1)

- The electro-active polymer energy conversion strength (B2)

The first phenomenon is a physical property based on the material used which cannot be modified after fabrication; however, variations can be imposed on the second phenomenon to damp more or less the system in order to maximize the energy absorption.

In order to increase the amount of energy extracted, the electro-active polymer rings need deformations with amplitudes of at least $10 \%$. Below this range, the available mechanical power that can be converted is lost into heat due to energy leakages. Moreover, the conversion of energy by the electroactive polymer rings damps out the system which in turn reduces the radial deformations. Hence, the maximum energy that can be extracted is a subtle balance between the tube radial deformations and the strength of the electro-active polymer rings for energy conversion.

Figure 6 shows the variation of the different powers depending on the energy extraction damping coefficient (B2). 


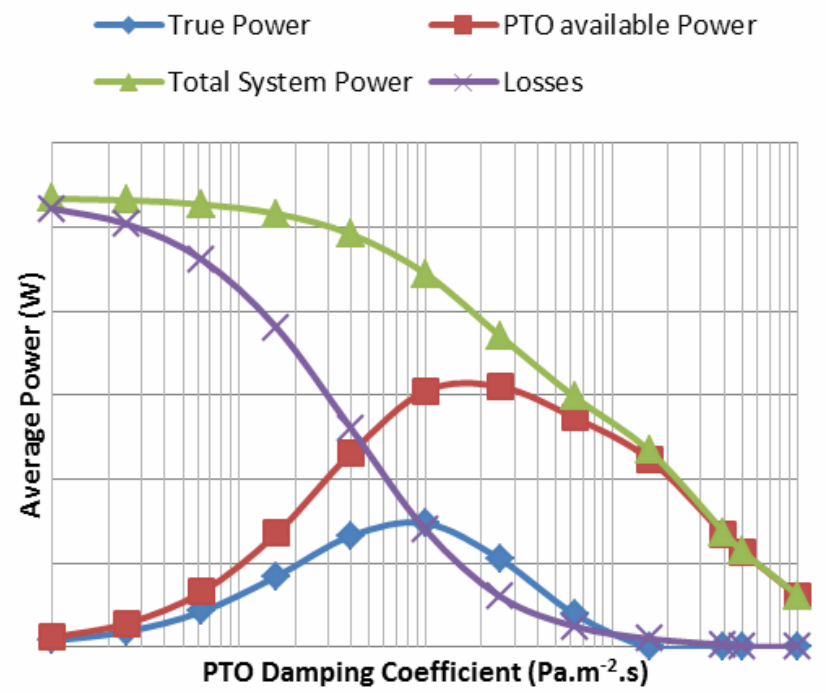

Figure 6. Evolution of the different average powers depending on the damping coefficient B2.

The $\mathrm{x}$ line corresponds to the energy losses due to the deformations of the elastomer. When the EAP rings are not activated, all the energy is converted into heat due to the viscous effects.

The triangles line corresponds to the total amount of energy available in the system. When the EAP rings are used, the radial deformations are inhibited leading to an overall decrease of the total energy.

The squares bell curve corresponds to the mechanical energy available for conversion by the EAP rings. The average mechanical energy increases proportionally with the damping coefficient B2. However, if this coefficient becomes too important, the system is inhibited and less energy is available for conversion.

The diamonds bell curve corresponds to the converted mechanical energy by the EAP rings. It follows the same pattern than the red curve and considers the deformation amplitudes that need to be at least above $10 \%$. This curve is lower than the available energy because of the energy leakages during the conversion. This represents a very rough estimation of the power electronics converters efficiency.

Once the damping coefficient for the EAP conversion has been tuned for maximum energy extraction, it is possible to estimate the fatigue life of EAP rings. The following relation gives a simple approach to the number of cycles allowed for a given deformation amplitude $\varepsilon$.

$$
N_{\text {break }}(\varepsilon)=a \varepsilon^{-b}
$$

The parameters $a$ and $b$ were established from tests carried out by SBM.
Once the extracted energy is known, with the fatigue life and the amount of required material for multiple configurations, it is possible to look for an optimal solution for a given deployment site

\subsection{Optimal configuration}

A parametrical approach is used in order to find an optimal configuration. Multiple tube geometries were generated with different length, diameter and wall thickness with the following intervals:

\section{- Length: $20 \mathrm{~m}$ to $500 \mathrm{~m}$}

- Inflated diameter: $0.4 \mathrm{~m}$ to $3 \mathrm{~m}$

- Wall thickness: $0.015 \mathrm{~m}$ to $0.09 \mathrm{~m}$

Every configuration was then evaluated with the following relation:

$$
N P V=-C A P E X+\sum_{1}^{N_{\text {yours }}} \frac{\text { Income }-O P E X}{(1+I R R)^{N_{\text {years }}}}
$$

The Net Present Value (NPV) is the remaining capital once the device has been exploited for $N_{\text {years }}$. For this simulation, the operational time has been set to 20 years.

The Internal Rate of Return (IRR) is a parameter to evaluate the desirability of a configuration. The higher the IRR, the more interesting a configuration is.

The Income is the capital gained from the production of energy. In this case the targeted mean annual power production is $400 \mathrm{~kW}$ for the wave climate of the EMEC test site in Scotland. It is considered that multiple tubes of the same configuration can be added together as an array to reach this target and have no influence over one another.

The investment costs (CAPEX) correspond to the material and mooring costs times the number of tubes in an array to reach $400 \mathrm{~kW}$. It is conservatively assumed that no gain can be made on shared mooring.

The operational costs (OPEX) correspond to the material costs if the WEC needs to be replaced during the 20 years of operation. Hence it depends on the lifetime of the specific configuration.

The optimal configuration is the one with a positive NPV and the highest IRR.

After using the previous relation on the different geometries, the optimal solution to generate $400 \mathrm{~kW}$ would be the following:

- $\quad$ Between 9 to 10 tubes

- Length of $290 \mathrm{~m}$

- Inflated diameter of $2.4 \mathrm{~m}$

- Thickness of $9 \mathrm{~cm}$

This implies that a long tube is more interesting than a short and very large tube. Indeed, theoretically, the available power inside the tube increases to the square of the diameter whereas it is only linearly proportional to the length.

The proposed solution can still be improved by increasing the number of initial geometries or by changing the algorithm. 


\section{CONCLUSIONS}

The tight collaboration between the LHEEA laboratory at Ecole Centrale de Nantes and the company SBM Offshore, in the context of the $\mathrm{S} 3$ project, financed by the agency ADEME, helped break through the first technological barriers required to design and optimize a revolutionary wave energy converter totally deformable and perfectly adapted to the complex nature of wave energy resources.

SBM now possesses a numerical tool experimentally calibrated to predict the production of energy of a deformable wave energy converter made of electro-active polymers. It is possible to optimize its different geometrical parameters and physical characteristics. This will help predict the technical specifications for the design of a prototype.

To bring this project up to an industrial scale, a technological leap will have to be done on the performance of electro-active polymers, their usage, fatigue life and their energy density.

\section{ACKNOWLEDGEMENTS}

The authors gratefully acknowledge the financial support from the French environmental agency ADEME through the funding scheme "Démonstrateurs en energies renouvelables et chimie verte" of Investissements d'Avenir (S3 Project). The authors would also like to thank the technical contributions from the staff at Hydrodynamic and Ocean Engineering Tank of Ecole Centrale De Nantes.

\section{REFERENCES}

[1] P. Jean, A. Wattez, G. Ardoise, C. Mélis, R. Van Kessel, A. Fourmon, E. Barrabino, J. Heemskerk, J.P. Queau, 2012, "Standing wave tube electro active polymer wave energy converter", in the SPIE Smart Structures/NDE conference, San Diego, California, USA.

[2] A. Babarit, J. Singh, C. Mélis, P. Jean. "A numerical model for analyzing the hydroelastic response of a flexible Electro Active Wave Energy Converter", Submitted for publication in Journal of Fluid and Structures, under revision.

[3] J.N. Newman, 1977, Marine Hydrodynamics, Cambridge, Massachussets: MIT Press.

[4] H. Bingham, 1999, "Computing the Green function for linear wave-body interaction", in the $13^{\text {th }}$ internation workshop on water waves and floating bodies, pp. 5-8.

[5] F.J.M. Farley, R.C.T. Rainey, J.R. Chaplin, 2012, "Rubber tubes in the Sea", Phil. Trans. R. Soc. A, 370: pp 381-402 